\title{
When is the price cost margin a safe way to measure changes in competition?*
}

\author{
Jan Boone $\dagger$ Jan C. van Ours ${ }^{\ddagger}$ and Henry van der Wiel ${ }^{\S}$
}

August 30, 2012

\begin{abstract}
The price cost margin $(\mathrm{PCM})$ is a popular way to measure competition. Although we know that this measure is not without problems, we actually do not know how often and under which conditions a change in PCM points in the wrong direction. We use a new competition measure, the profit elasticity (PE), which is more robust than PCM. Our empirical analysis based on Dutch data shows that when competition changes the probability that PCM points in the wrong direction increases with industry concentration.
\end{abstract}

Keywords: competition, profit elasticity, price cost margin, measures of competition, concentration

JEL classification: D43, L13

*This paper is a revised version of the paper previously entitled "How (not) to measure competition". Financial support from NWO (grant numbers 453-07-003 and 472.04.031) is gratefully acknowledged. The data analysis reported in this document was carried out at the Center for Policy Related Statistics of Statistics Netherlands. The views expressed in this document are those of the authors and do not necessarily reflect the policy of Statistics Netherlands. We thank Harold Creusen, Marc Duhamel, Free Huizinga, Lapo Filistrucchi, Maarten Pieter Schinkel, seminar participants at ACLE, CCP, Ente Einaudi, Industry Canada, NMa, University of Nottingham, participants at the NIE Christmas conference at UEA and two anonymous referees for useful comments and suggestions.

${ }^{\dagger}$ Department of Economics, Tilburg University, CentER, TILEC, IZA and CEPR. Email: j.boone@uvt.nl.

${ }^{\ddagger}$ Department of Economics, Tilburg University, Department of Economics, University of Melbourne, CentER, CESifo, CEPR and IZA. Email: vanours@uvt.nl.

$\S$ CPB. Email: hvdw@cpb.nl. 


\section{Introduction}

The price cost margin (PCM) has a long tradition as a measure of competition. There are papers (like Aghion, Bloom, Blundell, Griffith and Howitt (2005) and Nickell (1996)) that calculate PCM directly as the profits-sales ratio (we call this the "simple" PCM). Alternatively, with a structural approach, one first estimates demand and cost functions and then calculates the optimal PCM for each firm under an assumption on the relevant competitive model for the firms in the sector. By comparing a direct estimate of the PCM (like the profits-sales ratio) with the PCM predicted under different competitive regimes, one can identify which competitive regime applies in a sector. It is clear that the structural approach to empirical I.O. (see papers like Berry, Levinsohn and Pakes (1995), Bresnahan and Reiss (1991), Nevo (2001) and Reiss and Wolak (2007) for an overview) offers a better way to identify PCM and consequently firms' conduct. However, the instruments needed to estimate demand and cost relations are often missing in panel data sets with industry cross section. Indeed, the structural studies mentioned focus on one sector only. Hence they cannot identify the effects of competition on innovation, productivity etc. which are identified on differences across industries.

Conceptually, PCM has problems to pick up consistently the following two ways in which competition can be intensified in a market: (i) more firms in a market due to a fall in entry barriers and (ii) more aggressive conduct by incumbent firms. In the former case, more firms in the market reduce PCM in standard models. ${ }^{1}$ As we show below, in the latter case PCM can go up in response to an increase in competition intensity (incorrectly suggesting that competition went down).

A natural question then is: how often does the "simple" PCM measure point in the "wrong" direction? That is, how often does PCM fall (rise) while actually competition intensity went down (up)? This is the question

\footnotetext{
${ }^{1}$ Note that even this is not true for all demand and cost functions; see Amir and Lambson (2000) and Amir (2002) for details.
} 
we address in this paper. In order to do this, we need a competition measure which does not suffer from the biases present in PCM. As we show below, the profit elasticity $(\mathrm{PE})$ is such a robust competition measure.

We do not take a stand on the issue of how the PCM should be estimated. In the estimation of the $\mathrm{PE}$ measure, similar issues arise. In particular, one can chose a structural method to derive the demand and cost curves and then from these curves calculate the profit elasticity. However, because we want to understand how PCM performs in panel data sets with a cross section of industries, we use the direct method to estimate both PE and PCM. With this method we do not need additional information for all the markets in our sample, like cost instruments, product characteristics and instruments for consumers' taste parameters (such as demographic variables). Indeed, for most industries such data is not available. Therefore, we cannot use structural models to benchmark PCM. The new measure, PE, measures the percentage fall in profits due to a percentage increase in (marginal) costs. In any market, an increase in costs reduces a firm's profits. However, in a more competitive market, the same percentage increase in costs will lead to a bigger fall in profits. The underlying intuition is that in more competitive markets, firms are punished more harshly (in terms of profits) for being inefficient.

Using Dutch firm level data, we estimate both PCM and PE for 139 industries for the period 1993-2002. On average both measures point in the same direction. However, if the industry is concentrated, PCM can give the wrong impression about the change in intensity of competition: going up (down) over time while the intensity of competition went up (down). This is caused by the reallocation effect. As competition intensifies, market shares of efficient firms (with high price cost margin) increase at the expense of market shares of inefficient firms (with low price cost margins). This can raise the (weighted) industry PCM.

The implications of this observation are the following. If the data set used only has industry aggregate information on revenues and costs, PCM can be calculated and used safely for industries that are unconcentrated. For industry aggregate data sets there is usually information available about 
concentration in the industry (like a $\mathrm{C} 4$ or C5 ratio or a Herfindahl index that we use below). If the data set contains firm level information, it is better to estimate PE and use that as a competition measure, certainly for concentrated industries.

Papers like Boone, Griffith and Harrison (2005) and Creusen, Minne and van der Wiel (2006) also compare the PE and PCM measures using similar data as we do here. The contribution of our paper is to predict where PE and PCM point in opposite directions where the development of competition over time is concerned. Our model then suggests that in these cases of opposite predictions, PCM points in the wrong direction.

The set-up of our paper is as follows. Section 2 derives theoretically the properties of PCM and PE. Section 3 describes the data on competition measures and shows some key statistics. Section 4 shows that PE and PCM tend to deviate over time if the industry is concentrated. As shown in section 2 this is explained by the reallocation effect which causes PCM to go up (down) in response to an increase (fall) in competition intensity. In section 5 we report a number of robustness checks on how PE is estimated. The result that $\mathrm{PE}$ and $\mathrm{PCM}$ tend to deviate over time in concentrated industries remains true for the alternative specifications of PE. Section 6 concludes. Appendix A gives proofs of the results. Appendix B provides more details on how we constructed our data set.

\section{Model}

In this section, we briefly point out why from a theoretical point of view, PCM makes mistakes in concentrated markets. Then we derive the PE. 


\subsection{Price cost margin}

In empirical papers using panel data sets with a cross section over industries, PCM at the firm level is measured as

$$
\operatorname{pcm}\left(n_{i}, \theta\right)=\frac{R\left(n_{i}, \theta\right)-C\left(n_{i}, \theta\right)}{R\left(n_{i}, \theta\right)}
$$

where $n_{i}$ denotes firm $i$ 's efficiency level, $\theta$ captures the intensity of competition in $i$ 's industry, $R($.) denotes the firm's equilibrium revenue as a function of $n_{i}$ and $\theta$ and $C($.$) denotes the equilibrium variable costs of the firm. Equi-$ librium values for $p c m, R$ and $C$ also depend on $n_{-i}$ : vector of efficiency levels of $i$ 's competitors. To ease notation, this is suppressed when this does not cause confusion. Variable costs are costs that vary with the production level of the firm. In particular, fixed costs are not included in $C($.$) . Let c\left(q, n_{i}\right)$ denote the variable cost function of a firm with efficiency level $n_{i}$. Higher $n_{i}$ denotes higher efficiency in the sense that

$$
\frac{\partial c(q, n)}{\partial n} \leq 0, \frac{\partial^{2} c(q, n)}{\partial q \partial n}<0
$$

That is, higher efficiency (weakly) reduces the cost level $c$ and decreases marginal costs $\partial c / \partial q$. Let $q\left(n_{i}, \theta\right)$ denote the equilibrium output level of a firm with efficiency level $n_{i}$ in a market with competition intensity $\theta$. Then we have $C\left(n_{i}, \theta\right)=c\left(q\left(n_{i}, \theta\right), n_{i}\right)$.

Although below we argue that PCM is a problematic competition measure, throughout this paper we assume that at the firm level $\mathrm{pcm}$ is decreasing in competition intensity $\theta$. In particular, we make the following assumption.

\section{Assumption 1}

$$
\frac{\partial p c m\left(n_{i}, \theta\right)}{\partial n_{i}} \geq 0, \frac{\partial p c m\left(n_{i}, \theta\right)}{\partial \theta}<0, \frac{\partial^{2} \ln \left(p c m\left(n_{i}, \theta\right)\right)}{\partial n \partial \theta} \leq 0
$$

In words, more efficient firms, have higher $p c m$, which is intuitive. More efficient firms tend to price lower, but not to the extent that their $\mathrm{pcm}$ 
falls. Indeed in the data we find that more efficient firms have both higher profits and higher $p c m$ (as shown below, this follows from the fact that PE is positive). An increase in intensity of competition (higher $\theta$ ) reduces $\mathrm{pcm}$. Finally, although higher efficiency $n$ allows a firm higher $p \mathrm{~cm}$, the relative increase in $p c m$ (due to an increase in $n$ ) is dampened as competition becomes more intense.

To illustrate assumption 1, consider a simple example where $p c m=1 / \varepsilon$ where the elasticity $\varepsilon$ equals $\varepsilon=-\partial \ln q / \partial \ln p>0$. In a monopoly context, $\varepsilon$ is the market demand elasticity. In a oligopoly context, $\varepsilon$ denotes the firm's perceived demand elasticity (taking competitors' reactions into account). The first assumption implies that $\partial \varepsilon / \partial q \leq 0$ because more efficient firms tend to produce more: $\partial q / \partial n \geq 0$. The second assumption implies that an increase in $\theta$ raises the elasticity $\varepsilon$, that is $\partial \varepsilon / \partial \theta>0$. The final assumption can now be written as

$$
\frac{\partial^{2} \ln (p c m)}{\partial \theta \partial n}=\frac{1}{\varepsilon^{2}} \frac{\partial q}{\partial n}\left(\frac{\partial \varepsilon}{\partial q} \frac{\partial \varepsilon}{\partial \theta}-\varepsilon \frac{\partial^{2} \varepsilon}{\partial \theta \partial q}\right) \leq 0
$$

A sufficient condition for this to hold is that $\partial^{2} \varepsilon / \partial \theta \partial q \geq 0$. In words, although more efficient firms with higher output levels face a reduced elasticity, this reduction in $\varepsilon$ is smaller in more competitive environments. These are fairly standard assumptions and ensure that pcm at the firm level works fine.

To be able to analyze the intensity of competition at the industry level, firm level $p c m$ 's are aggregated into a weighted average industry PCM:

$$
\operatorname{PCM}(\theta)=\sum_{i \in I} s\left(n_{i}, \theta\right) \operatorname{pcm}\left(n_{i}, \theta\right)
$$

where $I$ denotes the set of firms in the market and $s($.$) denotes firm i$ 's market share:

$$
s\left(n_{i}, \theta\right)=\frac{R\left(n_{i}, \theta\right)}{\sum_{j \in I} R\left(n_{j}, \theta\right)}
$$

The advantage of defining $P C M$ like this, is that it can be derived from industry aggregate information on revenues and costs (i.e. without the need 
for firm level data):

$$
\operatorname{PCM}(\theta)=\frac{\sum_{i \in I} R\left(n_{i}, \theta\right)-\sum_{i \in I} C\left(n_{i}, \theta\right)}{\sum_{i \in I} R\left(n_{i}, \theta\right)}
$$

We analyze when this measure of industry intensity of competition correctly picks up changes in competition $\theta$ over time.

A change in competition intensity $\theta$ affects PCM in the following way

$$
\frac{d P C M}{d \theta}=\sum_{i \in I} \frac{d s\left(n_{i}, \theta\right)}{d \theta} p c m\left(n_{i}, \theta\right)+s\left(n_{i}, \theta\right) \frac{d p c m\left(n_{i}, \theta\right)}{d \theta}
$$

If all firms are symmetric $\left(n_{i}=n\right.$ for all $\left.i \in I\right)$ and no firm exits, market shares are not affected by $\theta$ and hence $d P C M / d \theta<0$ by assumption 1 . Further, if competition is intensified by an increase in the number of firms (due to a fall in entry barriers), firms' market shares fall and again we find $d P C M / d \theta<0$.

Now consider an increase in the variance of efficiency levels $n_{i}$, that is firms become asymmetric. This will increase concentration in the sector, as more efficient firms increase their market share at the expense of less efficient firms. Below we use the the Herfindahl index, defined as $H=\sum_{i \in I} s_{i}^{2}$, as a measure of concentration. It is straightforward to verify that an increase in the variance of $n_{i}$ (for given average efficiency) leads to higher $H$.

If $\theta$ is increased in the case with a positive variance in $n_{i}$, we get the following reallocation effect. As competition is intensified, market share is reallocated from inefficient to efficient firms. That is, $s_{i}$ increases for firms with a high $p c m_{i}$ and falls for firms with a low $p c m_{i}$. This raises the weighted average $P C M$ if the first term in (5) outweighs the second (negative) term. This is most likely to happen when the variance in $n_{i}$ is high and thus when concentration is high in the industry. It is straightforward to find theoretical examples where this indeed happens. The question we ask is: how often does this happen in real data?

In order to identify the reallocation effect in the data, we decompose the change in the PCM for a market in five different effects. This decomposition is 
reminiscent of the decomposition of the change in Total Factor Productivity as used by Baily, Hulten, Campbell, Bresnahan and Caves (1992) and Foster, Haltiwanger and Syverson (2008). In particular, we write

$$
\begin{array}{r}
P C M_{1}-P C M_{0}=\sum_{i \in I_{1}} s_{i 1} p c m_{i 1}-\sum_{i \in I_{0}} s_{i 0} p c m_{i 0}= \\
\sum_{i \in I}\{\underbrace{s_{i 0}\left(p c m_{i 1}-p c m_{i 0}\right)}_{\text {within effect }}+\underbrace{p c m_{i 0}\left(s_{i 1}-s_{i 0}\right)}_{\text {reallocation effect }}+\underbrace{\left(p c m_{i 1}-p c m_{i 0}\right)\left(s_{i 1}-s_{i 0}\right)}_{\text {interaction effect }}\} \\
+\underbrace{\sum_{i \in I_{1} \backslash I} s_{i 1} p c m_{i 1}}_{\text {entry effect }}-\underbrace{\sum_{i \in I_{0} \backslash I} s_{i 0} p c m_{i 0}}_{\text {exit effect }}
\end{array}
$$

where $I_{0}\left(I_{1}\right)$ is the set of active firms before (after) the change in competition, $I=I_{0} \bigcap I_{1}$ and $i \in I_{1} \backslash I$ if both $i \in I_{1}$ and $i \notin I$. In words, the set $I$ contains all firms that are active both before and after the increase in competition. Working with a balanced panel implies limiting the data to this set $I$. The set $I_{0} \backslash I\left(I_{1} \backslash I\right)$ contains firms that are active before the increase in competition but which are forced to exit after competition intensifies (firms that are active after the increase in competition but were not present before).

We use PE to identify when the reallocation effect causes the industry PCM to go up after an increase in competition. However, the discussion above suggests two other ways in which PCM can be (partly) corrected for this effect. We conclude this subsection with a discussion of both methods.

First, the reallocation effect can be partly eliminated by using the unweighted PCM as measure of competition (as in Aghion et al. (2005)). This reduces the problem caused by the reallocation effect to a certain extent (as shown in Boone, Griffith and Harrison (2005)) but does not remove it completely: an increase in competition tends to remove inefficient firms from the market with low PCM which raises the average PCM in the market. A disadvantage of the unweighted PCM is that the PCM of small firms gets a disproportionate effect on the industry aggregate PCM.

A second solution to the reallocation effect is to keep the weights $s_{i}$ fixed at their baseline values. In other words, one can use the within effect as 
a measure of competition. We do not take this approach for two reasons. First, the within effect has to be based on a balanced panel (the set $I$ in equation (6)). That is, if one wants to measure competition using the within effect consistently over a period of, say, 10 years one can only use data on the firms that are in the panel for all 10 periods. This limits the number of observations considerably if a data set is based on a (rotating) sample such as ours.2 Second, in our data the within effect is a magnitude 10 smaller than the entry and exit effects (due to the fact that we use an unbalanced panel). Hence due to the noise in the other effects, we cannot use the within effect in the data to benchmark PCM.

\subsection{Profit elasticity}

In order to identify when the PCM makes mistakes, we use PE. This is a robust competition measure closely related to the analysis in Boone (2008). This paper introduces a general model of competition where no assumptions are made on the functional form of demand and costs nor on the mode of competition. To illustrate, it is not necessary to assume homogeneous products nor linear demand or cost functions. Boone (2008) shows that for three efficiency levels $n_{1}>n_{2}>n_{3}$ it is the case that

$$
\frac{d\left(\frac{\pi\left(n_{1}, \theta\right)-\pi\left(n_{3}, \theta\right)}{\pi\left(n_{2}, \theta\right)-\pi\left(n_{3}, \theta\right)}\right)}{d \theta}>0
$$

where variable profits are defined as (using the notation introduced above) $\pi(n, \theta)=R(n, \theta)-C(n, \theta)$. This measure is called relative profits differences (RPD). Hence RPD is increasing in the intensity of competition, $\theta$. As shown in Boone (2008), this holds whether competition is intensified through entry (due to a fall in entry barriers) or through more aggressive conduct.

\footnotetext{
${ }^{2}$ Alternatively, one can calculate the within effect for consecutive years from $t$ to $t+1$ and then with a new sample from $t+1$ to $t+2$ etc. In this way, fewer observations are lost. The disadvantage of this approach is that the reallocation effect plays a role again in the comparison of competition between $t$ and $t+2$ as the base changes between those years. In this way, the within effect is not a consistent measure over the whole period. As explained below, PE is not affected by an unbalanced panel data set.
} 
To see the connection between PE and RPD, assume that we can approximate variable profits as follows:

$$
\ln \pi(n, \theta)=\alpha(\theta)-\beta(\theta) \phi(n, \theta)
$$

where we assume $\phi_{n}^{\prime}<0$. In words, $\phi$ tries to capture (marginal) costs, which are decreasing in efficiency. Below, we use log costs for $\phi$ and then $\beta$ equals PE.

First, assume that we can capture efficiency perfectly in the sense that $\phi(n, \theta)=\phi(n)$. In other words, our measure of marginal costs is not affected by intensity of competition $\theta$. We can show the following (proof in the appendix).

Proposition 1 Assume that $\phi(n, \theta)=\phi(n)$, then $\frac{d R P D}{d \theta}>0$ implies that $\frac{d \beta}{d \theta}>0$.

This result gives the intuition of the competition measure that we use in this paper. We estimate a relation between profits and costs as in equation (8). If we then observe that this relation becomes steeper over time (i.e. $\beta$ increases), we interpret this as more intense competition in the industry.

In the regressions below, we use the following cost measure: $\phi(n, \theta)=$ $\ln (C(n, \theta) / R(n, \theta))$. The advantage of using a logarithm here is that the slope $\beta$ in equation (8) can be interpreted as an elasticity. A side effect of using logarithms in equation (8) is that we have to delete observations with negative profits $(\pi<0)$ which could lead to a selection bias. As a robustness check van der Wiel (2010) estimated equation (8) with the left hand side replaced by $\ln ($ constant $+\pi)$ where the constant is high enough that no observations need to be deleted. The PE that follows from this estimation is highly correlated with the one that follows from equation (8) that we use here. Moreover, a recent Finnish study running similar regressions to ours (with and without the constant) finds that the two different PEs are strongly correlated in both levels and in first differences (Maliranta, Pajarinen, Rouvinen and Yl-Anttila (2007)). This suggests that ignoring loss-making firms does not generate a bias in the estimates of PE. 
Because we have no data on prices, we cannot derive cost per unit of output. Instead we use cost per unit of revenue. Because of assumption 1 we interpret firms with lower values for $\phi$ as being more efficient. The problem with this efficiency measure is that it is endogenous. In particular, changes in competition intensity $\theta$ affect our "independent variable" $\phi$ in regression equation (8). In appendix A we prove the following result.

Proposition 2 Under assumption 1, an increase (decrease) in $\beta$ can be interpreted as more (less) intense competition $\theta$.

In other words, although $\theta$ affects $\phi$ we can still interpret changes in the slope of equation (8) as changes in intensity of competition. As we cannot instrument $\phi$, there may be other endogeneity issues as well. As shown below, we test for these issues in the data and show that our results are not affected by it. This makes PE a robust competition measure. To illustrate, we are not aware of a similar, robust derivation of the relation between PCM and competition intensity.

In a sense, proposition 2 is more than we need. Even if assumption 1 would not hold, our results are still correct as long as the endogeneity issue with respect to cost measure $\phi$ is not correlated with concentration.

As shown by equation (9) below, when we estimate equation (8) firm and time fixed effects are included. These fixed effects capture variables (other than efficiency) that affect a firm's profits. In section $\underline{5}$, we do a robustness check with respect to the functional form of equation (8). Further, van der Wiel (2010) does a formal test on the functional form of the relation between profits and costs. In both cases, it turns out that equation (8) gives a good fit empirically. 


\section{Data on competition measures}

\subsection{Measuring PE}

Theory suggests that an increase in competition increases the slope $\beta$ in equation (8) with $\phi=\ln (C / R)$. In our analysis, we allow for the fact that we cannot perfectly observe the relevant values for firms' profits and marginal costs. For instance, a firm may produce other products than the products for the market under consideration. The statistical office (or other agency gathering the data) may decide to classify these sales and costs of other products under the same heading (industry classification). We denote the observed variable profit level for firm $i$ at time $t$ by $\pi_{i t} u_{i}$, where $\pi_{i t}=\pi\left(n_{i}, \theta_{t}\right)$. Hence the observation error equals $\left(u_{i}-1\right) \pi_{i t}$. Similarly, the observed costs are denoted by $\ln \left(\frac{C_{i t}}{R_{i t}} v_{i}\right)$. The assumption that we make is that these observation errors may differ between firms but are constant over time (or, if the observational errors do change over time, they change in the same way for all firms in a sector such that they are picked up by the time fixed effect).

Hence the version of equation (8) that we estimate is

$$
\ln \left(\pi_{i t} u_{i}\right)=\alpha_{t}-\beta_{t} \ln \left(\frac{C_{i t}}{R_{i t}} v_{i}\right)+\varepsilon_{i t}
$$

where $\alpha_{t}=\alpha\left(\theta_{t}\right)$ and $\beta_{t}=\beta\left(\theta_{t}\right)$. Or equivalently ${ }^{3}$

$$
\ln \left(\pi_{i t}\right)=\alpha_{i}+\alpha_{t}-\beta_{t} \ln \left(\frac{C_{i t}}{R_{i t}}\right)+\varepsilon_{i t}
$$

For each market, defined by a 3 digit SIC industry divided into SMEs (small and medium sized enterprises) and BEs (big enterprises) we estimate equation (10).

To control for changes of firms over time with respect to their SIC-code

\footnotetext{
${ }^{3}$ The firm fixed effect is given by $\alpha_{i} \approx-\ln \left(u_{i}\right)-\beta_{t} \ln \left(v_{i}\right)$. Note that the firm fixed effects are really fixed if $\beta_{t}=\beta$. We use an approximation which holds if the variation in $v_{i}$ is much bigger than the variation in $\beta_{t}$. The time fixed effect is given by $\alpha_{t}$.
} 
and their size class, firms are classified according to their SIC-code most reported and to their lowest size class level in the period observed.

Equation (10) contains constructed variables based on the same information on both the left and the right hand side. In particular, the profit variable on the left hand side is calculated as the difference between revenues and costs, the costs variable on the right hand side is calculated as the ratio of costs and revenues (to get a cost per unit variable). Measurement errors in firms' revenues or costs will tend to overemphasize the effects of costs on profits and hence PE can be biased. Nevertheless, as long as the errors are uncorrelated with a change in competition the change in $\mathrm{PE}$ will be measured correctly. Furthermore, to the extent that the measurement errors are time invariant they will be picked up by the firm fixed effects. 4 This also includes unobserved explanatory variables that are constant over time but may have an impact on profits. Although it is possible that the parameter estimates of PE are a mixture of "signal" and "noise", it is clear that the signal dominates as shown below. Finally, we report robustness checks in the estimation of PE and show that the results are unchanged.

\subsection{Comparing competition measures}

In this section we take a first look at the measures PE, Price Cost Margin and Herfindahl index based on Dutch firm level data from about 250 markets over the period 1993-2002. ${ }^{5}$ We define a market to be a 3-digit SIC-code divided

\footnotetext{
${ }^{4}$ Note that the potential bias introduced by the measurement errors may be corrected by the use of instrumental variables. Unfortunately, we do not have firm characteristics that could be used as instrumental variables.

${ }^{5}$ See for details appendix B. The calculation of PCM and $\mathrm{H}$ in the data is straightforward and has already been discussed in section 2. The top part of table 1 gives an overview of the two competition measures and H. Ideally, the number of observations should be $139 * 2 * 10=2780$ (i.e. 139 3-digit SIC-industries divided into SMEs and BEs for the period 1993-2002). However, the full sample contains less observations: 2104 observations. This smaller set is due to that (i) for manufacturing industries data only runs to 2001; (ii) not for every SIC-code SMEs or BEs are available; (iii) some SIC-codes are absent in particular years. Moreover, if we remove missing observations on the reallocation effect the sample is reduced to 1809 observations. Appendix B provides information about the distribution of the $t$-statistics. van der Wiel (2010) also considers subsamples where $\mathrm{PE}$ is estimated with at least $10 \%$ significance, where PE is restricted to be positive etc. Similar results to the ones reported here are found.
} 
into small and medium sized firms (which have less than 50 employees) and big enterprises (which have 50 employees and more).

Table 1 gives the summary statistics for the variables that we use in the analysis hereafter. Here, we work with the full sample of markets. We find that on average (over all markets and years) PE equals 6.9: a one percent increase in costs leads to a 6.9 percent reduction in profits. However, there is substantial variation in $\mathrm{PE}$. In one market, a one percent increase in a firm's costs leads to a 39 percent fall in its profits. In some markets the estimated $\mathrm{PE}$ is negative for some years (suggesting that higher costs lead to higher profits). Most of these (negative) estimates are not significantly different from zero. As reported by van der Wiel (2010), deleting such negative estimates of PE does not affect our results. The average values for PCM and $\mathrm{H}$ equal 0.18 and 0.12, respectively. Further, the standard deviations of both PCM and $\mathrm{H}$ are much smaller than the one for $\mathrm{PE}$. The higher variance in $\mathrm{PE}$ is not due to imprecision in estimating PE. As shown in figure 4, there are only a few market-time combinations with a t-value below 2 .

The variables $\triangle \mathrm{PE}$ and $\triangle \mathrm{PCM}$ denote first differences in PE and PCM. It is interesting to note that both variables are on average nearly zero. The bottom part of table 1 gives the decomposition of $\triangle \mathrm{PCM}$ using equation (6) for consecutive years (i.e. $T-1$ effects per industry). Of this decomposition we will later use the reallocation effect to identify cases where changes (over time) in PCM and PE contradict each other. As mentioned, due to our panel data set structure, the entry and exit effects are a factor ten bigger than the within and reallocation effects. If we would calculate the within effect using a balanced panel (to get a consistent measure over the 10 year period; not plagued by reallocation effects) we would lose $85 \%$ of our observations.

Figure 1a summarizes the PEs that we find in the data with histograms. We give separate histograms for the two sub-markets: small and medium sized firms and large firms. As one can see in this figure large firms have substantially higher values for PE than small and medium sized firms (which is the main motivation for us to subdivide markets in this way). This is in contrast to the idea in policy circles that entrepreneurs and small and 
Table 1: Overview of variables and decomposition of the $\triangle \mathrm{PCM}$

a. Summary statistics of variables

\begin{tabular}{rrrrr} 
Variable & Mean & Standard dev. & Minimum & Maximum \\
\hline $\mathrm{PE}$ & 6.91 & 5.13 & -5.47 & 39.07 \\
$\Delta \mathrm{PE}$ & -0.06 & 4.42 & -32.81 & 34.45 \\
$\mathrm{PCM}$ & 0.18 & 0.10 & 0.02 & 0.84 \\
$\Delta \mathrm{PCM}$ & 0.00 & 0.06 & -0.50 & 0.75 \\
$\mathrm{H}$ & 0.12 & 0.12 & 0.00 & 0.93
\end{tabular}

b. Decomposition $\triangle \mathrm{PCM}$ using equation (6)

\begin{tabular}{rrrrr} 
Effects & Mean & Standard dev. & Minimum & Maximum \\
\hline Within & 0.02 & 0.45 & -0.72 & 16.71 \\
Reallocation & 0.02 & 0.19 & -0.96 & 1.80 \\
Interaction & 0.01 & 0.11 & -0.62 & 3.01 \\
Entry & 0.27 & 0.35 & 0.00 & 6.08 \\
Exit & 0.26 & 0.20 & 0.00 & 0.99
\end{tabular}

Based on 1809 observations. Each of the decomposition effects is normalized by $P C M$ in the base (0) year. 
medium firms are key to economic performance. These firms supposedly increase productivity and competitiveness. In particular, these firms are claimed to face very intense competition and therefore have a big incentive to reduce costs and innovate. We find exactly the opposite. It is the big firms that face the higher values for PE. If their costs go up by $1 \%$ the percentage fall in profits is bigger. Note that this is not just a trivial size effect as we consider the percentage change in profits. $\cdot$

Figure 1b gives the histograms for PCM. PCM tends to be lower for large firms than for small and medium firms, again showing that large firms are active on a more competitive market. Our interpretation is that in many markets large firms compete on a national market while small and medium firms compete on local markets.

The histograms for $\mathrm{H}$ in figure $1 \mathrm{c}$ do not confirm the results seen for $\mathrm{PE}$ and PCM. The market for large firms tends to be more concentrated than the market for small and medium firms. Given that $\mathrm{H}$ is based on market shares, it is not surprising that large firms tend to be active on highly concentrated sub-markets. However, the histograms above for PE and PCM indicate that higher concentration should not (always) be associated with less intense competition.

\section{When is PCM correct?}

In this section, we address our main question: when we follow an industry over time, do PE and PCM tell the same story about the development of competition? To illustrate, if PCM increases over time, is it the case that PE falls (both signaling a fall in competition intensity)?

As shown in the top part of Table 1, the average changes in both PE and PCM are close to zero. Moreover, on average PCM and PE are negatively correlated (not reported separately). In other words, across industries and

\footnotetext{
${ }^{6}$ It is obviously the case that the absolute change in profits due to an increase in marginal costs is bigger for a firm with a higher output level.
} 
time periods, higher PE is associated with lower PCM; both measures indicating more intense competition. In this sense, both measures contain on average the same information about competition intensity.

However, this does not imply that PCM is always consistent with the robust measure PE. Within particular markets there may be changes over time which are not always mutually consistent in terms of changes over time in competition. It turns out that this type of inconsistency happens in roughly one third of the cases. ${ }^{7}$ To investigate this in more detail we first localize the markets in which there is an inconsistency between the two measures, i.e. markets where they are positively correlated from one period to the next. In these cases one measure indicates an increase in competition while the other measure indicates a decrease in competition intensity. We refer to these cases as being strictly inconsistent. However, if the changes in the measures are close to zero, the fact that they have similar signs does not matter that much. Such differences can be caused by observational errors and not by underlying changes in competition intensity. Only if both changes in the measures are substantially different from zero and with the same sign there is clearly something wrong. We focus on these cases in the following way.

We define a dummy variable $I_{z}$ which indicates whether or not $\triangle P E$ and $\triangle P C M$ are inconsistent, i.e. they have the same sign and are of sufficient magnitude. More specific we define $I_{z}=1$, if

$$
\triangle P E<\mu_{1, z} \text { and } \triangle P C M<\mu_{2, z}
$$

or

$$
\triangle P E>\mu_{1,100-z} \text { and } \triangle P C M>\mu_{2,100-z}
$$

and $I_{z}=0$ otherwise. Here $\mu_{1, z}$ is the value of the $z^{t h}$-percentile of the distribution of $\triangle P E$ and $\mu_{2, z}$ is the value of the $z^{t h}$-percentile of the distribution of $\triangle P C M$. Hence $I_{z}=1$ if both $\triangle P E$ and $\triangle P C M$ are either "strongly" negative or "strongly" positive. In these cases the two measures clearly con-

\footnotetext{
${ }^{7}$ Creusen, Minne and van der Wiel (2006) for the Netherlands and Boone, Griffith and Harrison (2005) for the UK also find such cases.
} 
tradict each other. This is illustrated by the areas labeled $A$ and $B$ in figure 2. Note that figure 2 is for illustration only. In the analysis reported below, areas $A$ and $B$ are much bigger -covering more observations- than suggested by the figure.

Now it is possible to investigate the determinants of the probability that the changes in the two measures are inconsistent, for various values of $z$. Figure 3 a shows how the probability of inconsistency increases with $\mathrm{H}$. This is hardly perceptible for cases with strict inconsistency $(z=50)$, but the increase is clear for low $z$ values, i.e. when there is a big inconsistency. Similarly, Figure $3 \mathrm{~b}$ shows that this probability of inconsistency is decreasing in the number of firms. Figure $3 \mathrm{c}$ shows that there is a non-linear relationship between the reallocation effect and the probability of inconsistency. Initially the probability of inconsistency goes down, later on it goes up. This is what one would expect: the reallocation effect distorts the relation between PCM and PE if it is either strongly negative (deciles 1 and 2) or strongly positive (deciles 9 and 10). When the reallocation effect is close to zero, it is unlikely to overturn the relation between PCM and PE. To capture this effect we use the absolute value of the reallocation effect. ${ }^{8}$

We investigate the determinants of inconsistency in more detail using a linear probability model to estimate the probability of inconsistency and relate this to $\mathrm{H}$, number of firms and the absolute value of the reallocation effect. Table 2 a presents the parameter estimates. Even if we consider the situation in which $\triangle \mathrm{PE}$ and $\triangle \mathrm{PCM}$ have the same sign - the changes are strictly inconsistent $-\mathrm{H}$ has a positive and significant effect (although this is not visible in figure $3 \mathrm{a}$ ). Table $2 \mathrm{~b}$ adds the absolute size of the reallocation effect and the number of firms (in logs) to the explanatory variables. The

\footnotetext{
${ }^{8}$ Note that the effect we describe in section 2.1 is indeed the reallocation effect (not the interaction effect): an increase in competition can raise the market share of existing firms with high pcm relative to existing firms with low pcm. Further, the entry/exit effects that we calculate in the data do not necessarily correspond to entry/exit in the market. To illustrate, in our data a firm may "enter" because it is sampled this year and not the previous year (where it existed as well). Finally, the reallocation effect can also be identified in balanced data sets. Although this is not an issue in our data set, this may be useful for other researchers who want to do a similar analysis.
} 
number of firms in the market has a negative and significant effect on the probability of inconsistency for values of $z$ below 45. Intuitively, with many small firms in the market, the reallocation effect will not be big enough to push PCM in the "wrong" direction. Further, the reallocation effect is significant for low values of $z$. In markets with a high $\mathrm{H}$, a low number of firms and a big reallocation effect we find that the probability of inconsistency between PCM and PE is large.

We conclude that the reallocation effect is responsible for the inconsistency between the changes in PE and PCM over time. There is direct evidence because the probability of inconsistency increases with the reallocation effect. For this effect to be significant, we need to focus more on the tails of the distributions of $\triangle \mathrm{PE}$ and $\triangle \mathrm{PCM}(z=35)$. There is also indirect evidence because the probability of inconsistency increases with $\mathrm{H}$ and falls with the number of firms.

\section{Robustness checks}

To investigate the robustness of our estimation results we run four alternative equations compared to our basic equation (10) $\cdot$ ?

The first alternative way to estimate PE is that we switch places for the dependent variable and the explanatory variable. In fact, this is one way to test the impact of measurement problems

$$
\ln \left(\frac{C_{i t}}{R_{i t}}\right)=\alpha_{i}+\alpha_{t}-\tilde{\beta}_{t} \ln \left(\pi_{i t}\right)+\varepsilon_{i t}
$$

In this case, $\mathrm{PE}$ is defined as $P E_{t}=1 / \tilde{\beta}_{t}$

The second alternative allows for a non-linear relationship between $\ln \left(\pi_{i}\right)$

\footnotetext{
${ }^{9}$ More details can be found in van der Wiel (2010), which examines a number of ways to estimate PE and analyzes the estimation results for a number of subsamples.
} 
Table 2: Probability of inconsistency between $\triangle P E$ and $\triangle P C M$; parameter estimates linear probability model

\begin{tabular}{|c|c|c|c|c|}
\hline & H-index & $\begin{array}{c}\text { Reallocation } \\
\text { effect }\end{array}$ & $\begin{array}{l}\text { Number } \\
\text { firms }\end{array}$ & $\begin{array}{l}\text { Percentage } \\
\text { inconsistent }\end{array}$ \\
\hline \multicolumn{5}{|c|}{ a. Baseline estimates $-\mathrm{I}$} \\
\hline 1. $z=50$ & $0.14(1.6)$ & & & 45.6 \\
\hline 2. $z=45$ & $0.32(3.1)^{* *}$ & & & 36.3 \\
\hline 3. $z=40$ & $0.44(4.4)^{* *}$ & & & 28.0 \\
\hline 4. $z=35$ & $0.50(5.6)^{* *}$ & & & 20.3 \\
\hline \multicolumn{5}{|c|}{ b. Baseline estimates - II } \\
\hline 1. $z=50$ & $0.07(0.6)$ & $0.10(1.0)$ & $-0.07(0.9)$ & 45.6 \\
\hline 2. $z=45$ & $0.11(0.9)$ & $0.12(1.3)$ & $-0.19(2.3)^{* *}$ & 36.3 \\
\hline 3. $z=40$ & $0.19(1.7)^{*}$ & $0.13(1.5)$ & $-0.24(3.1)^{* *}$ & 28.0 \\
\hline 4. $z=35$ & $0.26(2.6)^{* *}$ & $0.21(2.5)^{* *}$ & $-0.22(3.5)^{* *}$ & 20.3 \\
\hline \multicolumn{5}{|c|}{ c. Alternative estimates $(z=35)-\mathrm{I}$} \\
\hline Baseline & $0.50(5.6)^{* *}$ & & & 20.3 \\
\hline Alternative 1 & $0.66(5.9)^{* *}$ & & & 20.3 \\
\hline Alternative 2 & $0.74(7.1)^{* *}$ & & & 29.4 \\
\hline Alternative 3 & $0.52(4.8)^{* *}$ & & & 22.3 \\
\hline Alternative 4 & $0.64(6.6)^{* *}$ & & & 27.4 \\
\hline \multicolumn{5}{|c|}{ d. Alternative estimates $(z=35)-$ II } \\
\hline Baseline & $0.26(2.6)^{* *}$ & $0.21(2.5)^{* *}$ & $-0.22(3.5)^{* *}$ & 20.3 \\
\hline Alternative 1 & $0.52(3.8)^{* *}$ & $0.10(1.4)$ & $-0.13(2.0)^{* *}$ & 20.3 \\
\hline Alternative 2 & $0.17(1.3)$ & $0.10(1.1)$ & $-0.56(8.6)^{* *}$ & 29.4 \\
\hline Alternative 3 & $0.19(1.4)$ & $0.11(1.3)$ & $-0.28(3.9)^{* *}$ & 22.3 \\
\hline Alternative 4 & $0.31(2.6)^{* *}$ & $0.18(2.1)^{* *}$ & $-0.31(4.4)^{* *}$ & 27.4 \\
\hline
\end{tabular}

Note: Reallocation effect in absolute terms, number of firms in logs divided by 10; based on 1809 observations except for the Alternative 3 estimates (1740 observations); all estimates contain calendar year fixed effects; absolute $t$-statistics corrected for clustering of observations across markets in parentheses; a ** $(*)$ indicates a parameter estimate significantly different from zero at a $5 \%(10 \%)$ level. 
and $\ln \left(C_{i} / R_{i}\right)$ :

$$
\ln \left(\pi_{i t}\right)=\alpha_{i}+\alpha_{t}-\beta_{1 t} \ln \left(\frac{C_{i t}}{R_{i t}}\right)+\beta_{2 t}\left(\ln \left(\frac{C_{i t}}{R_{i t}}\right)\right)^{2}+\varepsilon_{i t}
$$

Due to this non-linearity, the results for the $\beta$ 's cannot be directly interpreted as a measure of market competition. Taking the first derivative of (14) with respect to $\ln (C / R)$, we get

$$
\frac{\partial \ln \left(\pi_{i t}\right)}{\partial \ln \left(\frac{C_{i t}}{R_{i t}}\right)}=-\beta_{1 t}+2 \beta_{2 t} \ln \left(\frac{C_{i t}}{R_{i t}}\right)
$$

which is different for different firms in the market. A market value for PE can now be derived by using the market average of the marginal cost $\left(\overline{C_{i t} / R_{i t}}\right)$ as follows: $P E_{t}=-\beta_{1 t}+2 \beta_{2 t} \ln \left(\overline{C_{i t} / R_{i t}}\right)$.

The third alternative measure for $\mathrm{PE}$ is that we use a balanced panel instead of an unbalanced panel to make sure that our results are not driven by panel attrition. To be left with sufficient observations, we use a balanced panel for two subperiods: 1993-1997 and 1998-onwards respectively.

The fourth alternative is that we adjust the cost concept accounting only for the labor costs and neglecting the costs for materials and other intermediate inputs. This relaxes the problem of using the same variables to construct the left hand side and right hand side of equation (10). Table $2 \mathrm{c}$ and $2 \mathrm{~d}$ checks whether our main result is robust to these alternative specifications of PE.

We find for all four alternatives that the probability of inconsistency is higher in more concentrated markets (higher $\mathrm{H}$ and lower number of firms). Our main result in section 4 is robust to different ways in which PE can be estimated. 


\section{Conclusion}

There are a number of questions for which one needs a competition measure that varies both over time and across industries. Examples we have in mind include the relation between competition on the one hand and variables like innovation, unemployment, wages, price inflation on the other hand.

A structural econometrics approach to identify firms' conduct in a market is then not an option (because good instruments are missing for most industries). Well known studies on the relation between competition and innovation rely on a PCM measure that can be derived from industry aggregate data on revenue and variable costs. We derive a robust competition measure, $\mathrm{PE}$ - the percentage fall in profits due to a $1 \%$ increase in (marginal) costs - to benchmark PCM. We find that the industry aggregate PCM performs well in unconcentrated industries. In concentrated industries an increase in competition intensity can raise PCM (due to the reallocation effect) making it less reliable.

Hence if only industry aggregate data is available, PCM can be used without problems for industries with low concentration. For concentrated industries firm level data is needed. In that case, we would advise to estimate PE. Although one can correct PCM for the reallocation effect, these corrections are either imperfect or lead to a substantial reduction in the number of observations. PE is a robust way to determine the intensity of competition in a market. In this paper, we have used a direct way to estimate PE without using instruments. A structural approach to estimating PE is left for future research.

The firm level data set that we use here, is collected by Statistics Netherlands to publish the national accounts. Such data is increasingly available in many countries, which makes it possible to do international comparisons of the development of competition at the sector level. To facilitate the comparisons of $\mathrm{PE}$ in different countries, it would be useful if at OECD level the following variables are measured in the same way: revenue out of the sale of products (not including, say, the sale of a business unit) and variable costs 
like blue collar labour, intermediate inputs and energy. In order to calculate costs per unit of output as an efficiency measure, a measure of output would simplify the estimation of PE.

Due to confidentiality restrictions on the data, it is impossible for a researcher to pool firm level data from different countries. Hence a further step would be if national statistical offices would report estimated PEs (per year for each industry) to a supra-national statistical office like Eurostat. 


\section{References}

Aghion, P., N. Bloom, R. Blundell, R. Griffith and P. Howitt. 2005. "Competition and innovation: an inverted U relationship." Quarterly Journal of Economics CXX(2):701-728.

Amir, R. 2002. "Market structure, scale economies and industry performance." mimeo.

Amir, R. and V. Lambson. 2000. "On the effects of entry in Cournot markets." Review of Economic Studies 67(2):235-254.

Baily, M.N., C. Hulten, D. Campbell, T. Bresnahan and R. Caves. 1992. "Productivity dynamics in manufacturing plants." Brookings Papers on Economic Activity. Microeconomics pp. 187-267.

Berry, S., J. Levinsohn and A. Pakes. 1995. "Automobile prices in market equilibrium." Econometrica 63:841-890.

Boone, J. 2008. "A new way to measure competition." Economic Journal 118:1245-1261.

Boone, J., R. Griffith and R. Harrison. 2005. "Measuring competition." AIM Working Paper no. 22.

Bresnahan, T.F. and P.C. Reiss. 1991. "Entry and competition in concentrated markets." Journal of Political Economy 99(5):977-1009.

Creusen, H., B. Minne and H. van der Wiel. 2006. "Competition in the Netherlands; an analysis of the period 1993-2001." CPB Document No. 136.

Foster, L., J. Haltiwanger and C. Syverson. 2008. "Reallocation, firm turnover and efficiency: selection on productivity or profitability." American Economic Review 98(1):394-425.

Maliranta, Mika, Mika Pajarinen, Petri Rouvinen and Pekka Yl-Anttila. 2007. COMPETITION IN Finland: TRENDS ACROSS BUSINESS SECTORS IN 1994-2004. KTM Julkaisuja 13 ETLA.

Nevo, A. 2001. "Measuring market power in the ready-to-eat cereal industry." Econometrica 69:307-342.

Nickell, S. 1996. "Competition and Corporate Performance." Journal of Political Economy 104:724-746. 
Reiss, P. and F. Wolak. 2007. Handbook of Econometrics. Vol. 6A Elsevier Science Publishers chapter Structural econometric modeling: Rationales and examples from industrial organization.

van der Wiel, Henry. 2010. Competition and innovation: Together a tricky rollercoaster for productivity $\mathrm{PhD}$ thesis Tilburg University. 


\section{Appendix A. Proof of Results}

Proof of proposition 1 With the profit function as specified in equation (8), we can write

$$
R P D=\frac{e^{\beta\left(\phi_{3}-\phi_{1}\right)}-1}{e^{\beta\left(\phi_{3}-\phi_{2}\right)}-1}
$$

where $\phi_{i}=\phi\left(n_{i}\right)$. It follows that

$$
\frac{d R P D}{d \theta}=\frac{d R P D}{d \beta} \frac{d \beta}{d \theta}>0
$$

where the equality follows from the assumption that $\phi($.$) does not depend$ on $\theta$ and the inequality follows from equation (7). Hence a necessary and sufficient condition for $\frac{d \beta}{d \theta}>0$ is $\frac{d R P D}{d \beta}>0$ or equivalently

$$
\frac{\phi_{3}-\phi_{1}}{1-e^{-\beta\left(\phi_{3}-\phi_{1}\right)}}>\frac{\phi_{3}-\phi_{2}}{1-e^{-\beta\left(\phi_{3}-\phi_{2}\right)}}
$$

This is true because $n_{1}>n_{2}>n_{3}$ implies (by assumption 1) $\phi_{3}-\phi_{1}>\phi_{3}-\phi_{2}$ and it is the case that

$$
\frac{d\left(\frac{x}{1-e^{-\beta x}}\right)}{d x}>0 . \underline{10}
$$

Q.E.D.

Proof of proposition 2 Using equation (7), we know that

$$
\frac{d R P D}{d \theta}=\frac{d R P D}{d \beta} \frac{d \beta}{d \theta}+R P D\left[\frac{d \ln R P D}{d\left(\phi_{3}-\phi_{1}\right)} \frac{d\left(\phi_{3}-\phi_{1}\right)}{d \theta}+\frac{d \ln R P D}{d\left(\phi_{3}-\phi_{2}\right)} \frac{d\left(\phi_{3}-\phi_{2}\right)}{d \theta}\right]>0
$$

Hence, if the expression in square brackets is non-negative, we can conclude from an observed increase in $\beta$ that $R P D$ increased. That is, an increase in $\beta$ in this case implies an increase in $\theta$.

The remainder of this proof shows that assumption 1 implies that the expression in square brackets is non-negative. Equivalently, (since $\phi_{3}-\phi_{1}>$

\footnotetext{
${ }^{10}$ To see this, note that the inequality can be written as
}

$$
\frac{e^{\beta x}-1-\beta x}{\left(1-e^{-\beta x}\right)^{2}}>0
$$


$\left.\phi_{3}-\phi_{2}\right)$ we need to show that for given $\beta$ it is the case that

$$
\frac{d^{2} \ln \left(e^{\beta\left(\phi_{3}-\phi_{1}\right)}-1\right)}{d \theta d\left(\phi_{3}-\phi_{1}\right)} \geq 0 .
$$

This condition can also be written as

$$
\frac{d}{d \theta}\left(\frac{\beta e^{\beta\left(\phi_{3}-\phi_{1}\right)}}{e^{\beta\left(\phi_{3}-\phi_{1}\right)}-1}\right) \geq 0
$$

This is satisfied if $\frac{d\left(\phi_{3}-\phi_{1}\right)}{d \theta} \leq 0$ or equivalently if

$$
\frac{d^{2} \phi(n, \theta)}{d \theta d n} \geq 0
$$

To see that this is implied by assumption 1, we introduce the following notation: $R_{i}=\left(1+\mu_{i}\right) C_{i}$. Then $p c m_{i}=\mu_{i} /\left(1+\mu_{i}\right)$ and assumption 1 can be written as

$$
\begin{aligned}
\mu_{n_{i}}^{\prime} & <0 \\
\mu_{\theta}^{\prime} & <0 \\
\frac{\partial^{2} \ln \left(p c m\left(n_{i}, \theta\right)\right)}{\partial n \partial \theta} & =\frac{1}{\mu(1+\mu)}\left[\mu_{n_{i} \theta}^{\prime \prime}-\mu_{n}^{\prime} \mu_{\theta}^{\prime}\left(\frac{1}{\mu} \frac{1}{1+\mu}\right)\right] \leq 0
\end{aligned}
$$

With $\phi=\ln (C / R)=-\ln (1+\mu)$ we can write condition (A.5) as follows

$$
\begin{aligned}
\frac{\partial^{2} \phi(n, \theta)}{\partial n \partial \theta} & =\frac{-1}{1+\mu}\left[\mu_{n \theta}^{\prime \prime}-\frac{1}{1+\mu} \mu_{n}^{\prime} \mu_{\theta}^{\prime}\right] \\
& >\frac{-1}{1+\mu}\left[\mu_{n \theta}^{\prime \prime}-\left(\frac{1}{1+\mu}+\frac{1}{\mu}\right) \mu_{n}^{\prime} \mu_{\theta}^{\prime}\right] \\
& \geq 0
\end{aligned}
$$

where the first inequality follows from equations (A.6), (A.7) and the second from (A.8). Hence, indeed, we have $\partial^{2} \phi / \partial n \partial \theta \geq 0$ as required by (A.5).

Q.E.D. 


\section{Appendix B. Data and measurement issues}

\section{Initial uncleaned data set}

The estimates for PE, PCM and H are based on firm-level data for the Netherlands. These data are derived from the annual survey for the "Production Statistics" (PS) by Statistics Netherlands. The survey gives complete coverage of firms with at least 20 employees, while firms with fewer than 20 employees are sampled. This paper focuses on the period 1993-2001 (and 2002 for service industries). The data set has been constructed after matching the detailed accounting data over time. We have no data at our disposal on the agriculture and fishing industry, banking and insurance, public utilities and health care industries but otherwise we cover all industries in the Netherlands.

It turns out that the matched data set was not complete for all industries in manufacturing and wholesale trade. For some industries at the 3 digit SIC code, observations for certain years were missing for firms with size less than 100 employees. Therefore, we excluded all observations of these industries. Furthermore, for the analysis of the competition measures, we leave out firms without employees.

\section{From uncleaned to cleaned data set}

Unprocessed firm-level data may contain errors for various reasons. In order to obtain reliable firm-level data we performed several 'cleaning' activities (largely similar to Creusen, Minne and van der Wiel (2006)). We removed: 1) observations of firms with no turnover and employment; 2) the second observation of the same firm in one year; 3 ) observation of year $t+1$ if a firm has identical output and employment data (or value added) in two consecutive years; 4) observation of firms with negative variable profits; 5) observations of firms with negative intermediate inputs; 6 ) observations of firms with huge changes in key variables as output and employment; in particular, firms with more than $500 \%$ increase in turnover or employment or decrease by more than $80 \%$ in these variables. Finally, due to confidentiality requirements of Statistics Netherlands, we had to remove 3-digit SIC industries if less than 5 firms per year were available. The table below shows that the consequences of all those cleaning steps are limited:

$\begin{array}{lcc}\text { Variable } & \text { Uncleaned dataset } & \text { Cleaned dataset } \\ \text { Number of firms } & 288660 & 236346 \\ \text { Average firm size sample } & 71 & 74 \\ \text { Number of workers (x1000) } & 27559 & 23718 \\ \text { PCM } & 0.16 & 0.18\end{array}$


All in all, approximately 52000 firms (i.e approximately 18 percent) have been removed from the matched data set to obtain a clean data set for further analysis. This cleaned data set contains almost 240,000 observations over the period 1993-2002 based on information of about 90,000 different firms in the Netherlands from 139 industries at the 3-digit SIC-level.

Basic variables

To measure the three measures H, PCM and PE, we use a number of variables. All variables are based on the PS. Gross output denotes the value of output of firms including other activities (e.g. industrial services such as installation costs). Labor costs are defined as the salary of employees including social security charges and extra allowances. Intermediate inputs consist of costs like materials, energy and marketing. The variable costs are calculated as the sum of the labor costs and the intermediate inputs. Because data on marginal costs are absent, we use the variable costs over gross output (average variable costs, AVC) as an approximation. Profits $(\pi)$ are defined as firm's revenue (or gross output) minus variable costs. The definitions of $\mathrm{PCM}$ and $\mathrm{H}$ are discussed in the main text.

\section{Precision of PE-estimates}

Figure 4 shows the distribution of the $t$ statistics for the estimates of PE. The average value of the $t$ statistics is equal to 6.5 , the minimum value is equal to -1.2 , the maximum value is equal to 35.1. There are 6 observations for which the $t$ statistic is smaller than zero and of the positive $t$ statistics there are 196 which have a value below 1.96, the 5\%-critical value.

Properties of PE

To illustrate the properties of $\mathrm{PE}$, we perform a number of regressions in which the $\mathrm{PE}$ in market $k$ at time $t$ is explained through a number of market characteristics that are assumed to be exogenous to competition.

$$
P E_{k t}=\gamma_{0}+\gamma_{t}+x_{k t}^{\prime} \gamma+\epsilon_{k t}
$$

where $x$ is a vector of market characteristics, the $\gamma^{\prime} s$ are parameters - with $\gamma_{t}$ being calendar year fixed effects ${ }^{11}$ - and $\epsilon$ is an error term. As market characteristics we use the labor share in value added, the import share, the type of industry (dummy variable for manufacturing) and firm size (dummy variable for large firms).

We view these market characteristics as exogenous. Nonetheless, we acknowledge that the first two characteristics are not completely exogenous to

\footnotetext{
${ }^{11}$ The calendar year fixed effects are included to take cyclical effects into account.
} 
Table 3: Properties of PE

\begin{tabular}{lccc}
\hline & $(1)$ & $(2)$ & $(3)$ \\
Labor Income Share & $13.24(10.3)^{* *}$ & $15.81(12.9)^{* *}$ & $12.49(8.5)^{* *}$ \\
Import share & $0.74(0.8)$ & $2.12(0.6)$ & $3.56(1.0)$ \\
Large firms & $1.91(5.8)^{* *}$ & $2.06(11.1)^{* *}$ & - \\
Manufacturing & $3.98(8.2)^{* *}$ & - & - \\
Fixed effects & - & 137 & 245 \\
\hline
\end{tabular}

Note: Based on 1809 observations; all estimates contain fixed effects for calender years; column (2) contains fixed effects for each SBI, column (3) contains fixed effects for each market (SBI * firm size); absolute $t$-statistics in parentheses, in column (1) corrected for clustering of observations across markets; a ** $\left(^{*}\right)$ indicates a parameter estimate significantly different from zero at a $5 \%(10 \%)$ level.

the intensity of competition. To illustrate, intensity of competition in the product market can affect labor unions' bargaining power, thereby affecting the wage rate and the labor income share. Further, domestic markets where firms hardly compete are particularly attractive for foreign firms to enter, leading to a high import share. These caveats should be kept in mind. However, we believe that both variables are also driven by exogenous variation. The market's technology determines how much capital is needed to produce thereby affecting the capital income share and its complement the labor income share. Also, some products are easier to import than others which affects the import share. Markets where foreign products are close substitutes of domestic firms' products will face a tougher competitive regime. It is this effect that we try to capture.

Table 3 shows the parameter estimates. The labor income share has a positive effect on PE. A high labor income share indicates low capital costs and hence it is easier to enter the market. The import share has a positive but insignificant effect on PE. The dummy variable for manufacturing industries also has a positive and significant effect on PE. Conditional on the other market characteristics there is more competition in manufacturing industries than in service industries. Also in markets where large firms operate there is more competition. If we introduce SBI fixed effects or market fixed effects the effect of the labor income share on PE remains highly significant and positive. 

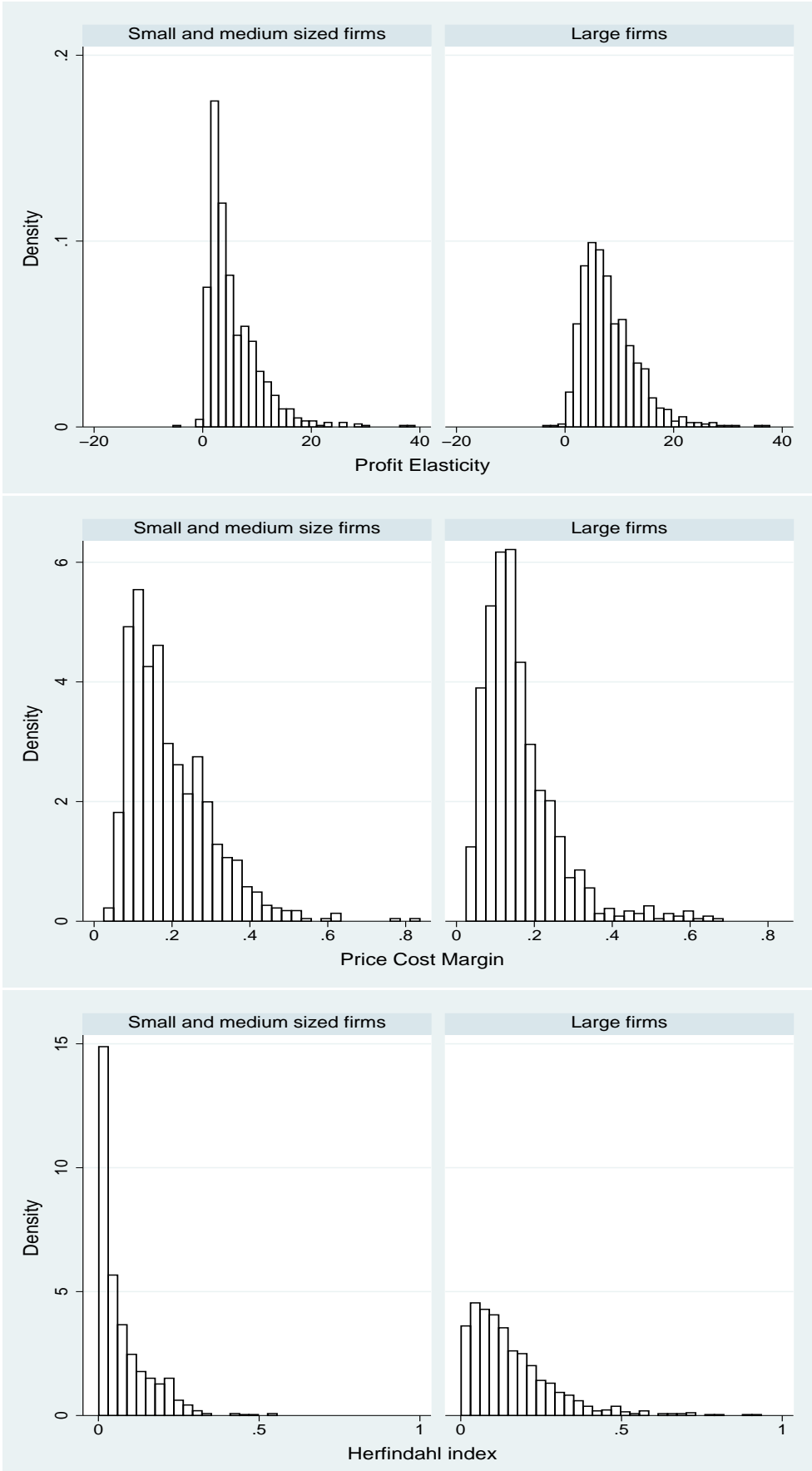

Figure 1: Distribution of PE, PCM, and $\mathrm{H}$ in the Dutch economy 
Figure 2: $\triangle P E$ and $\triangle P C M$ are "very" inconsistent for market-year combinations in areas $A$ and $B$.

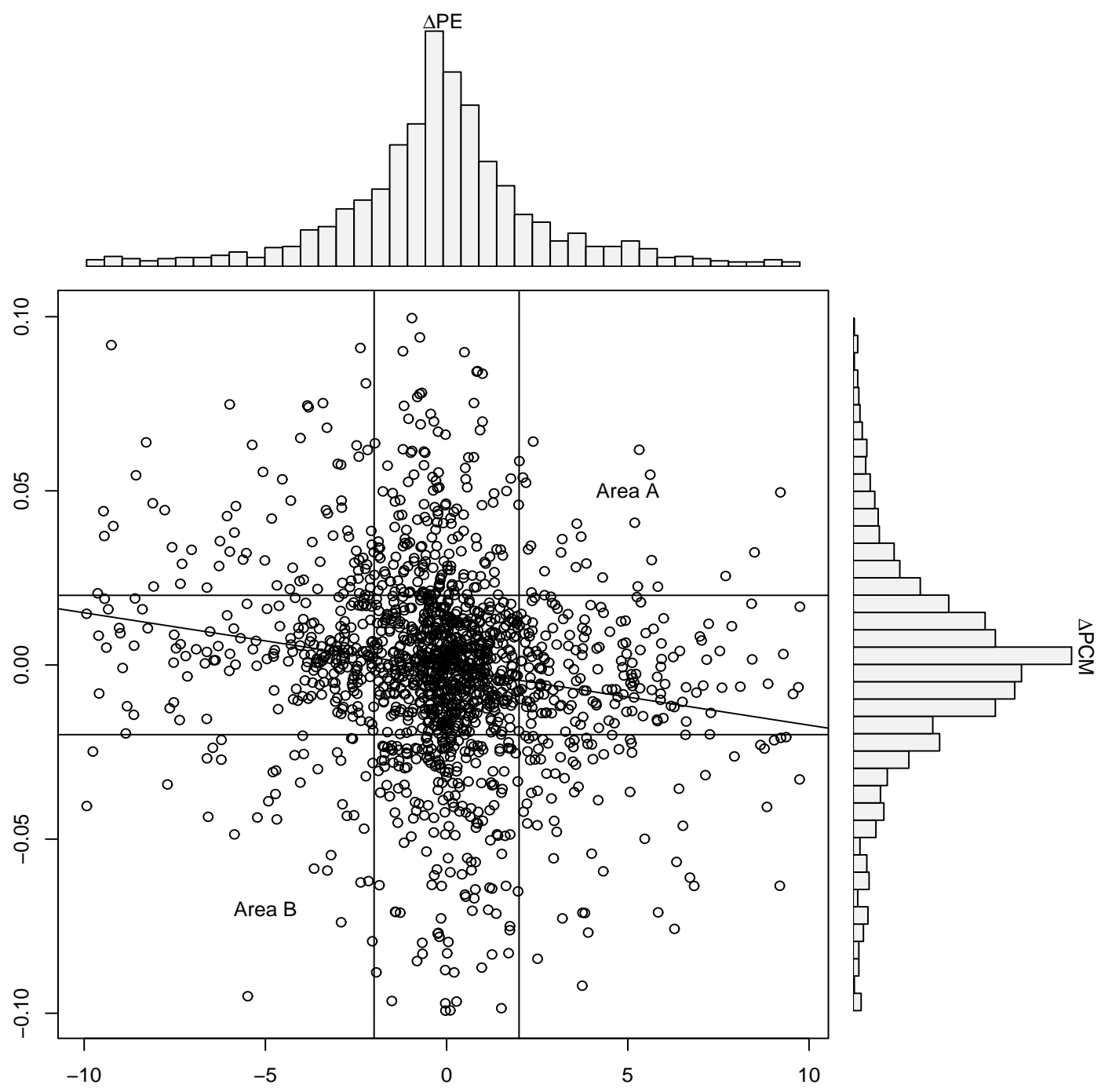



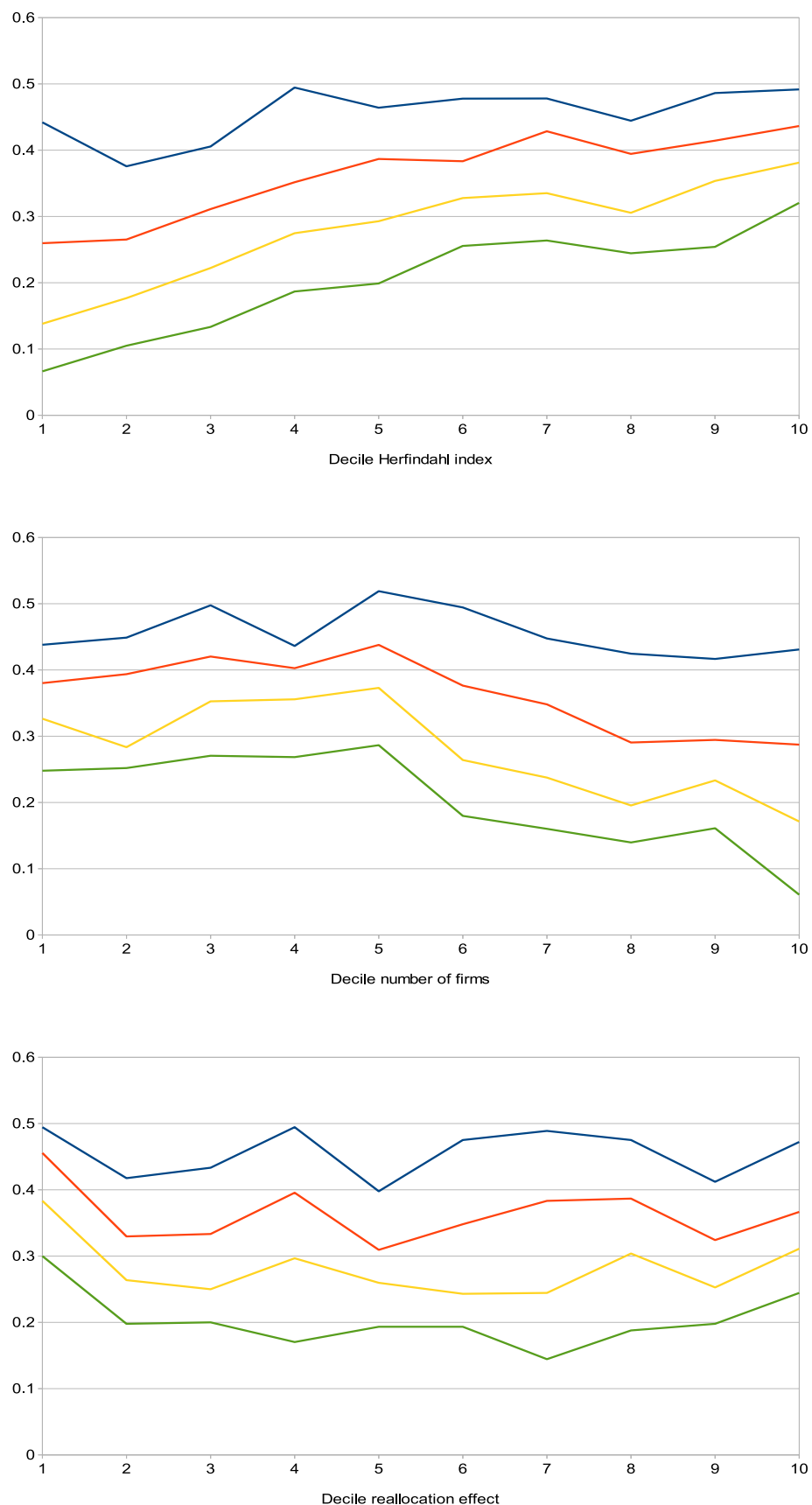

Figure 3: Probability of inconsistency between PE and the Price Cost Margin by value of $z$ and as a function of deciles of (a) Herfindahl index (top graph), (b) number of firms in the market (middle graph), (c) reallocation effect (bottom graph).

Note that the ordering of the lines is by value of $z$, from top to bottom: $z=50, z=45, z=40, z=35$. 


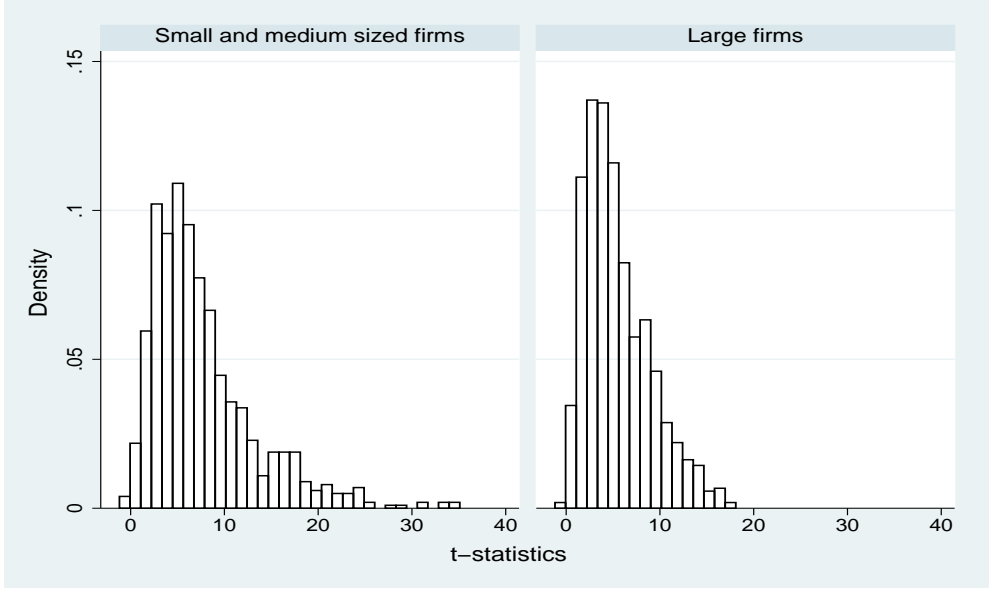

Figure 4: Distribution of $t$-values of the estimated PE 


\section{University Library}

\section{- M M N E R VA A gateway to Melbourne's research publications}

Minerva Access is the Institutional Repository of The University of Melbourne

Author/s:

Boone, J;van Ours, JC; van der Wiel, H

Title:

When is the Price Cost Margin a Safe Way to Measure Changes in Competition?

Date:

2013-03-01

Citation:

Boone, J., van Ours, J. C. \& van der Wiel, H. (2013). When is the Price Cost Margin a Safe Way to Measure Changes in Competition?. ECONOMIST-NETHERLANDS, 161 (1), pp.45-67. https://doi.org/10.1007/s10645-012-9196-7.

Persistent Link:

http://hdl.handle.net/11343/282534 